\title{
AUTOMATED LOW-COST PHOTOGRAMMETRY FOR FLEXIBLE STRUCTURE MONITORING
}

\author{
Chih-Heng Wang, Jon P. Mills*, Pauline E. Miller \\ School of Civil Engineering and Geosciences, Newcastle University, Newcastle upon Tyne, NE1 7RU, U.K. \\ (chih-heng.wang, jon.mills, pauline.miller)@ncl.ac.uk
}

Commission V/5

KEY WORDS: Close range photogrammetry, deformation, structural monitoring, mobile device

\begin{abstract}
:
Structural monitoring requires instruments which can provide high precision and accuracy, reliable measurements at good temporal resolution and rapid processing speeds. Long-term campaigns and flexible structures are regarded as two of the most challenging subjects in monitoring engineering structures. Long-term monitoring in civil engineering is generally considered to be labourintensive and financially expensive and it can take significant effort to arrange the necessary human resources, transportation and equipment maintenance. When dealing with flexible structure monitoring, it is of paramount importance that any monitoring equipment used is able to carry out rapid sampling. Low cost, automated, photogrammetric techniques therefore have the potential to become routinely viable for monitoring non-rigid structures.
\end{abstract}

This research aims to provide a photogrammetric solution for long-term flexible structural monitoring purposes. The automated approach was achieved using low-cost imaging devices (mobile phones) to replace traditional image acquisition stations and substantially reduce the equipment costs. A self-programmed software package was developed to deal with the hardware-software integration and system operation. In order to evaluate the performance of this low-cost monitoring system, a shaking table experiment was undertaken. Different network configurations and target sizes were used to determine the best configuration. A large quantity of image data was captured by four DSLR cameras and four mobile phone cameras respectively. These image data were processed using photogrammetric techniques to calculate the final results for the system evaluation.

\section{INTRODUCTION}

With the development of engineering techniques, designs of constructions have become increasingly complex (Glisic et al., 2007). How to comprehensively estimate a structural state and detect deformation using more effective and economic methods has become one of the most concerning issues for engineers in recent times (Kim et al., 2006). Measurements using GPS network, total station and terrestrial laser scanner, etc. have therefore been widely applied for structural health monitoring purposes. These instruments can provide accurate measurements from millimetre to micrometre levels and most of these instruments are largely operated automatically (GonzálezAguilera et al., 2009; Lin et al., 2008; Psimoulis and Stiros, 2007). However, when dealing with a long-term monitoring task, measurements based on these instruments may not be suitable for long period, in-situ applications due to the price issue, maintenance and the risk of theft or damage.

Photogrammetry is a non-contact measurement technique which utilises images to make accurate 3D measurements of complex objects (Luhmann, 2007). With fast sampling, wide coverage, photogrammetry is therefore likely to become one of the most important methods for flexible structural monitoring in engineering (Maas, 2006); combined with remote control techniques and the use of small volume and inexpensive imaging devices, a photogrammetric monitoring system can be placed in test fields for long-term monitoring purposes.

\footnotetext{
* Corresponding author
}

This research aims to develop a photogrammetric monitoring system which meets the following criteria:

- $\quad$ suitable for application to various types of structures;

- $\quad$ the ability to deal with long-term monitoring tasks;

- allows users to remotely control the monitoring system;

- fully automated operational processing;

- a cost-effective monitoring solution.

In this research, programmable mobile phones (also known as smartphones) were used to replace the conventional imaging instruments more usually adopted in general photogrammetric applications. Although the functionality of a smartphone can be significantly expanded by installing different types of application software (an app), there are no 'apps' currently available for structural monitoring or photogrammetric purposes. The main core of the low-cost, mobile phone-based monitoring system developed here is a self-programmed integrated software package. This software allows the operator to remotely control multiple mobile phones in order to conduct long-term monitoring tasks remotely instead of operating those instruments directly in the test field. Most of the embedded operations have been designed to automate processing, thus minimising human interaction and effort. Several tests have been undertaken to estimate the performance of this low-cost monitoring system. 
In this experiment, a shaking table was used as a test object to simulate a flexible structure. This experiment lasted for four days; during which time, four mobile phone camera stations were used to simultaneously capture imagery that could be used to examine the performance and remote control capabilities of the system. Compared with mobile phone built-in cameras, DSLR cameras have a better image sensor quality, resolution, lens resolving power, etc. and these features may affect the accuracy of photogrammetric measurements significantly (Akca and Gruen, 2009). In addition to the use of mobile phone cameras, four DSLR cameras were therefore also applied to provide a benchmark for comparison.

\section{CHARACTERISTIC OF EXPERIMENTAL INSTRUMENTS}

\subsection{Software development}

The mobile phone software development performed in this research was based on the Google Android OS 2.2 platform due to the following reasons:

- It allows users to implement multitasking;

- It supports the Java programming language which is a high interoperability programming language adapted for each OS;

- The source code and software development kit are free and open source.

At the time of carrying out this research, the Google Android $\mathrm{OS}$ is the most appropriate mobile platform to develop the software package for this experiment, as it provides a relatively open system for developers. However, this may change in future with the continued evolution of mobile phone technology.

\subsection{Experimental software}

The aim of this research was to design a low-cost, straightforward monitoring system. In order to achieve this goal, the integration of commercial off-the-shelf components (hardware and software) was essential. Meanwhile, the use of unique instruments must be avoided. As the instruments applied in this research were not customised for structural monitoring purposes, the integration of the various system components became the biggest challenge. A self-programmed software package was developed in order to control the mobile phone devices remotely. This software package was programmed using Java Platform, Enterprise Edition (Java EE) 6. The software was divided into three parts; a control platform which can be run from a PC or laptop interface; the other two were designed to install into mobile phone operating system (OS) to be responsible for dealing with commands which were sent from a terminal controller (a laptop or a PC). By installing the software into a mobile phone, the mobile phone can be treated as an automated, in-situ photogrammetric workstation; multiple tasks can be performed at the same time, such as receiving commands from a terminal computer, adjusting the photographic parameters, capturing a sequence of photographs, and saving previous image data, etc. Another module under development aims to provide a portable measurement function, allowing an operator to implement basic photogrammetric surveying in the test field by using one single mobile phone.
The photogrammetric processing software used in this research was PhotoModeler Scanner Version 6. This is commercial, offthe-shelf software. According to the user manual, PhotoModeler claims that when using a high quality digital camera, accuracies of 1:30,000 can be achieved (Kim et al., 2006). Previous research has validated PhotoModeler against another photogrammetric software package, The Vision Metrology System (VMS) (Wang et al., 2010), confirming that this commercial software is capable of meeting the demands of this project. Given its relatively low cost and user-friendly graphical user interface (GUI), PhotoModeler has become a popular choice for reverse engineering and many other engineering applications (Young and Garde, 2007).

\section{METHODOLOGY}

\subsection{System integration}

In laboratory testing, a shaking table experiment was undertaken to simulate a flexible structure for monitoring purposes. This shaking table $(1.4 \mathrm{~m} \times 1.4 \mathrm{~m} \times 0.1 \mathrm{~m})$ was driven by the Instron ${ }^{\circledR} 3520$ series hydraulic power units. The movements of this shaking table were directly recorded by a personal computer (Instron, 2011). Four DSLR cameras were configured to provide coverage of the shaking table, and their shutters were controlled with cable releases. The cable releases were connected to the same PC that controls the shaking table. The PC controls the photographic parameters (for example, shutter speed, camera ISO and exposure interval etc.) of the four DSLR cameras as well as the amplitude and frequency of the shaking table. The time lag of exposures from each DSLR camera can be recorded to the PC, the average time lag between the individual DSLR exposures being less than 5 microseconds. The PC was also connected to a laptop by means of a switch box. The laptop was used to remotely control four mobile phone cameras via the in-house software. This software programme can adjust the mobile phone cameras' photographic parameters via a $3 \mathrm{G}$ or a Wi-Fi network. Through the switch box, the PC can also be synchronized with the laptop via this software. Specifically, the information (shutter speed, exposure epoch and exposure interval etc.) of the four mobile phone cameras can be sent back to the PC from the laptop, then can be combined with the data from the shaking table and the DSLR cameras to carry out a comprehensive comparison.

The four mobile phones used were HTC Incredible S / 8megapixel with a built-in $4.57 \mathrm{~mm}$ f2.4 lens. The four DSLR cameras that were used to create a photogrammetric benchmark for comparison were Canon 50D / 15-megapixel with nominal $28 \mathrm{~mm}$ f2.8 lens (see Table 1).

\begin{tabular}{|c|c|c|}
\hline & HTC Incredible S & Canon 50D \\
\hline Image format & $3264 \times 2448$ & $4752 \times 3168$ \\
& 8-megapixel & 15 -megapixel \\
\hline Sensor size & $4.6 \times 3.4 \mathrm{~mm}$ & $22.3 \times 14.9 \mathrm{~mm}$ \\
\hline Pixel size & 1.4 microns & 4.7 microns \\
\hline Manual focus & Yes & Yes \\
\hline Output format & JPEG \& TIFF & JPEG, TIFF \& RAW \\
\hline
\end{tabular}

Table 1: Technical specifications of the cameras 


\subsection{In-house software}

A major goal for developing a controller software package is to build a bridge for communication between a terminal controller and one or more mobile devices. With the use of the software developed herein, operators can send commands to trigger multiple mobile phone-based camera stations simultaneously and periodically via $3 \mathrm{G}$ or wireless local area networks (WLAN). In addition, this software can access each mobile phone and sort image data following an exposure epoch. These data are stored temporarily in each mobile phone and are sent back to the terminal computer when the whole imaging sequence is finished. An overview of the software functions and operational processes are illustrated in Figure 1.

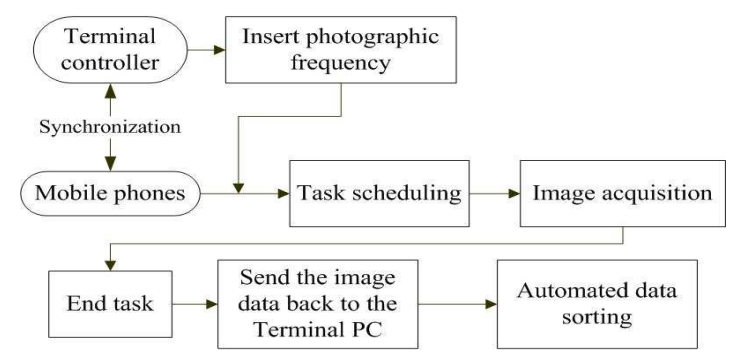

Figure 1: Operational processes of in-house software.

As this software was designed for long-term monitoring purposes, the state of each mobile device is automatically monitored by the computer to ensure each camera station is functioning correctly.

\subsection{Arrangement of test site}

During a four-day experiment, eight imaging stations (including four DSLR cameras and four mobile phone cameras) were arranged in two different locations to provide convergent coverage of the shaking table, and to evaluate the effect of different network configuration on photogrammetric precision. The network geometry configuration is illustrated in Figure 2 and shown in Figure 3.

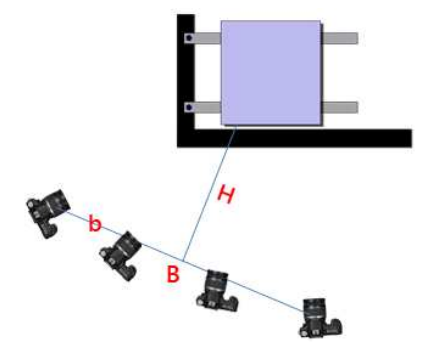

Figure 2: Shaking table test. B is the longest distance between two cameras; $b$ is the baseline between any two adjacent cameras and $\mathrm{H}$ is the object distance, as measured from the shaking table to the midpoint of $\mathrm{B}$.

The test was performed over a duration of four days. On the first day, the camera stations were arranged relatively close to the shaking table, with $\mathrm{H}$ at approximately $2.5 \mathrm{~m}$; whilst the value of B was $1.8 \mathrm{~m}$ (b was $0.6 \mathrm{~m}$ ). This configuration was unaltered for day 2 and day 3. On the fourth day, the values of both $\mathrm{H}$ and $\mathrm{B}$ were increased to $3.0 \mathrm{~m}$ in both cases, with camera convergence altered accordingly.
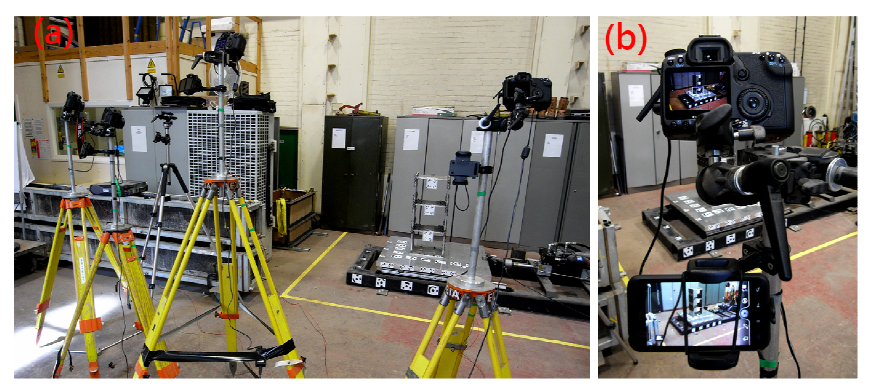

Figure 3: (a) Experimental configuration. (b) A close-up photograph of an individual image station; the mobile phones were mounted on customized tripod mounts.

\subsection{Mobile phone synchronization}

In order to ensure that the mobile phone cameras were triggered simultaneously, a high accuracy of synchronization was essential. To achieve this, all mobile phones were calibrated with respect to an established benchmark. The NTP (Network Time Protocol) is a TCP/IP protocol for synchronising time over a network. A client can request the current time from a local server, and uses the up-to-date information from NTP to set its own clock (Mills, 1991). In this research, NTP was chosen as a benchmark due to its high precision (within 10 milliseconds over the public internet and 0.2 milliseconds precision in a local area network (Mills, 2006)). A program was developed and applied to the mobile phones in order to refresh their internal clocks by updating the information from the NTP server every five minutes.

\subsection{Image acquisition procedure}

The frequency of exposure of the DSLR cameras was set to 10 seconds. The state of each camera was monitored by a computer. After every exposure, an electronic pulse was sent back to the terminal computer from each DSLR camera. The computer checked this information automatically to ensure all cameras were firing simultaneously. The frequency of exposure for the mobile phones was three times slower (30 seconds per frame) due to their slower memory buffer. During the test, the multiple DSLR camera stations were launched first, and the time of exposures were sent back to the PC. Then, this information was transferred to the laptop via a switch box. In accordance with the exposure epochs of the DSLR cameras, the self-programmed software synchronized the mobile phone stations via WLAN, sending a script which included the frequency of exposure and the start time for the first exposure (corresponding to the DSLR camera stations). Following this, the mobile phone cameras fired at the 30 second frequency until the operator pressed the stop button.

\subsection{System stability}

The shaking table test lasted for four days. On the first and the last days the flexible structure monitoring experiment was performed. The DSLR camera stations and the mobile phone stations were continuously capturing images of the moving table during the period of the experiment, at the frequencies detailed in the previous section. The DSLR camera stations were turned off when the shaking table was stopped. However, the four mobile phone cameras continued capturing images during the middle two days in order to test the stability of this low-cost system for long-term applications. Over the middle days, the four mobile phones were still monitored by the self- 
programmed software which was installed on the laptop. The system automatically rebooted all mobile phone stations and reassigned new tasks to each station if the software detected that any one of the mobile phones suffered a malfunction or had become unresponsive.

\subsection{Validation of photogrammetric measurements}

Photogrammetry is a non-contact measurement technique, the deformation of target structure can be calculated by analysing the movement of targets that were attached onto the structure's surface in advance (Luhmann, 2007). The use of light sensitive reflection targets, high definition target shapes, and good configuration of targets and imaging stations, etc. can improve the photogrammetric results significantly (Ganci and Clement, 2000). The target issue was examined in this experiment using different sizes of targets and two different configurations of imaging station layout.

The photogrammetric targets were made of two layers of selfadhesive vinyl sheets (Figure 4). These used a series of coded targets provided by commercial photogrammetric software, PhotoModeler Scanner Version 6 (herein subsequently referred to as PhotoModeler).

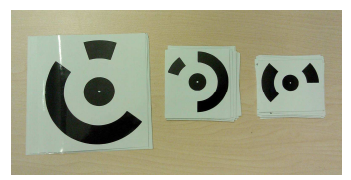

Figure 4: Customized target stickers with different target sizes.

Four different sized targets were applied to estimate the effect on the accuracy. The largest target size (the diameter of the central dot) was $16 \mathrm{~mm}$ whilst the other three sizes were $12 \mathrm{~mm}$, $9 \mathrm{~mm}$ and $4 \mathrm{~mm}$, respectively. These targets were evenly distributed across the shaking table surface with the configuration of coded targets as shown in Figure 5. Twentynine of forty-four targets were attached onto the platform facade the other fifteen targets were placed on a flexible structure $(0.3 \mathrm{~m} \times 0.3 \mathrm{~m} \times 0.7 \mathrm{~m})$ with the bottom being fixed onto the shaking table platform. Furthermore, there were nine extra coded targets arranged on the static external framework to provide stable results to compare with the dynamic targets that were arranged on the shaking table.

To facilitate rigorous evaluation of the system performance, a physical measurement was used as a benchmark to compare with the photogrammetric results. The thirty-eight targets that were attached onto solid parts of the shaking table (twenty-nine targets on the platform surface and nine targets on the external framework) were measured in three dimensions by a Leica total station (TCRP 1201 which has an angle measurement precision of 1") using spatial intersection. Ten point-to-point distances (Figure 5) were then calculated based on the coordinate data and used as a physical comparison to the photogrammetric data. The endpoints of these measurements included different target sizes in order to estimate their relative precisions. The two endpoints of the scaling distance shown in Figure 5 used $9 \mathrm{~mm}$ coded targets. The orange measurements used $4 \mathrm{~mm}$ targets as endpoints, whilst the green measurements used $16 \mathrm{~mm}$ targets. The blue lines represented measurements with $9 \mathrm{~mm}$ target endpoints and the single pink line in the middle of the platform used $12 \mathrm{~mm}$ coded targets as endpoints. The validation of the photogrammetric measurements was made by comparing these physical measurements with the photogrammetric results. Deviations from the physical measurements were calculated for statistical analysis.

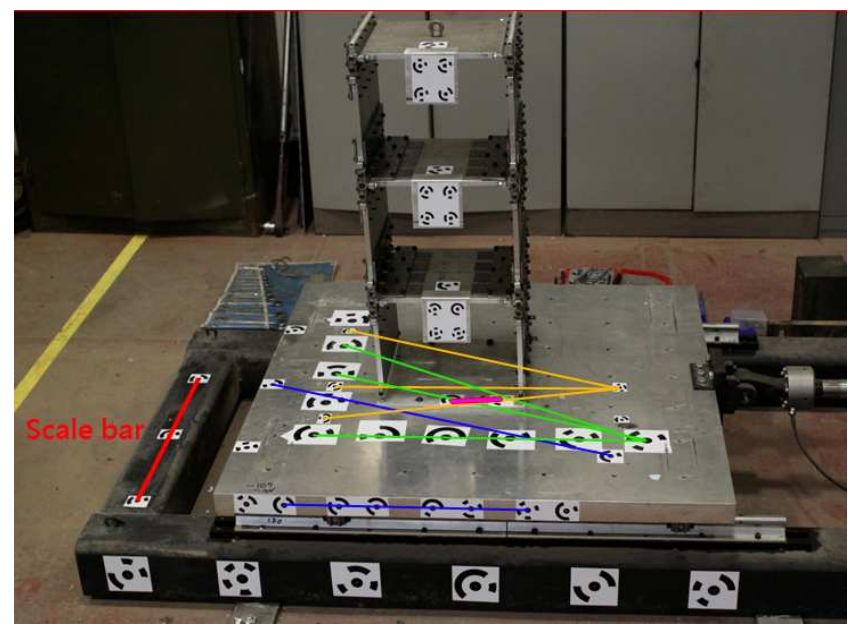

Figure 5: Selected measurements for validation comparison.

\section{RESULTS}

\subsection{Automated system recovery}

During the two days of the stability tasking (no movement), each mobile phone station should have generated at least 576 photographs (in this static object sampling period, the frame rate was downgraded from 30 seconds / per frame to 5 minutes / per frame). However, the number of photographs actually produced from each station was less than 576 due to individual system crashes. Table 2 shows the total number of images produced by each mobile phone station and also details the number of times the system crashed.

\begin{tabular}{|c|c|c|c|c|}
\hline & Station 1 & Station 2 & Station 3 & Station 4 \\
\hline Total images (frames) & 563 & 563 & 563 & 563 \\
\hline Numbers of crash & 3 & 4 & 4 & 2 \\
\hline
\end{tabular}

Table 2: Details of mobile camera image station frame capture over the stability monitoring period.

According to the information in Table 2, the four mobile phone cameras captured the same quantity of images which means that the four cameras had the same number of shutter actuations. Each camera captured 563 images and the photographs from each camera were automatically sorted to 563 folders by the self-programmed software. Each folder included 4 photographs from four different cameras for the same exposure epoch. The EXIF (Exchangeable Image File Format) data of each picture was then exported and the exposure epochs were checked. After careful inspection, it was determined that all photographs in the same folders had exactly the same exposure epoch.

\subsection{Comparison of photogrammetric results and physical measurements}

In this section, the precision of the monitoring system was comprehensively evaluated. Table 3 shows diameters corresponding to different target sizes on the photographs. 


\begin{tabular}{|c|c|c|c|c|}
\hline \multirow{2}{*}{$\begin{array}{c}\text { Target } \\
\text { size }\end{array}$} & \multicolumn{2}{|c|}{ DSLR Camera } & \multicolumn{2}{c|}{ Mobile Phone Camera } \\
\cline { 2 - 5 } & $\mathrm{B}=1.8 \mathrm{~m}$ & $\mathrm{~B}=3.0 \mathrm{~m}$ & $\mathrm{~B}=1.8 \mathrm{~m}$ & $\mathrm{~B}=3.0 \mathrm{~m}$ \\
& $\mathrm{H}=2.5 \mathrm{~m}$ & $\mathrm{H}=3.0 \mathrm{~m}$ & $\mathrm{H}=2.5 \mathrm{~m}$ & $\mathrm{H}=3.0 \mathrm{~m}$ \\
\hline $16 \mathrm{~mm}$ & 56 pixels & 50 pixels & 29 pixels & 24 pixels \\
\hline $12 \mathrm{~mm}$ & 40 pixels & 36 pixels & 18 pixels & 14 pixels \\
\hline $9 \mathrm{~mm}$ & 32 pixels & 26 pixels & 12 pixels & 9 pixels \\
\hline $4 \mathrm{~mm}$ & 19 pixels & 13 pixels & 9 pixels & 6 pixels \\
\hline
\end{tabular}

Table 3: Estimated diameter of target centres under different network configurations.

PhotoModeler recommends that centre target widths in the photos be at least 8-10 pixels wide (Kim et al., 2006). The results in Table 3 indicate that $9 \mathrm{~mm}$ and $4 \mathrm{~mm}$ targets may not have been big enough when the object distance was increased from $2.5 \mathrm{~m}$ to $3.0 \mathrm{~m}$. Following Foss (2008), the minimum target size using PhotoModeler software can be determined as follow:

Min. target centre dot diameter $=(10 \times \mathrm{fw} \times \mathrm{D}) /(\mathrm{f} \times \mathrm{pw})$

Where $f$ is the focal length, $f w$ is the format width of the camera, $D$ is the distance between the farthest target and a camera position, and $p w$ is the number of pixels in the width of the image.

\begin{tabular}{|c|c|}
\hline \multicolumn{2}{|c|}{ DSLR Camera } \\
\hline $\mathrm{B}=1.8 \mathrm{~m} ; \mathrm{H}=2.5 \mathrm{~m}$ & Min. Target Size $=4 \mathrm{~mm}$ \\
\hline $\mathrm{B}=3.0 \mathrm{~m} ; \mathrm{H}=3.0 \mathrm{~m}$ & Min. Target Size $=6 \mathrm{~mm}$ \\
\hline \multicolumn{2}{|c|}{ Mobile Phone Camera } \\
\hline $\mathrm{B}=1.8 \mathrm{~m} ; \mathrm{H}=2.5 \mathrm{~m}$ & Min. Target Size $=7 \mathrm{~mm}$ \\
\hline $\mathrm{B}=3.0 \mathrm{~m}, \mathrm{H}=3.0 \mathrm{~m}$ & Min. Target Size $=10 \mathrm{~mm}$ \\
\hline
\end{tabular}

Table 4: Minimum target diameters for different configurations.

Table 4 details the recommended minimum target size (centre dot diameter) following Equation (1). The results are largely a function of the object distance, and when compared to the outcomes in Table 3 , it is evident that the $4 \mathrm{~mm}$ and $9 \mathrm{~mm}$ targets may not be suitable for application when the camera to object distance is $3.0 \mathrm{~m}$.

\begin{tabular}{|c|l|l|l|l|l|l|}
\hline DSLR & \multicolumn{3}{|c|}{$\mathrm{B}=1.8 \mathrm{~m}, \mathrm{H}=2.5 \mathrm{~m}$} & \multicolumn{3}{c|}{$\mathrm{B}=3.0 \mathrm{~m}, \mathrm{H}=3.0 \mathrm{~m}$} \\
\hline & $\begin{array}{c}\sigma \mathrm{x} \\
(\mu \mathrm{m})\end{array}$ & $\begin{array}{c}\sigma \mathrm{y} \\
(\mu \mathrm{m})\end{array}$ & $\begin{array}{c}\sigma \mathrm{z} \\
(\mu \mathrm{m})\end{array}$ & $\begin{array}{c}\sigma \mathrm{x} \\
(\mu \mathrm{m})\end{array}$ & $\begin{array}{c}\sigma \mathrm{y} \\
(\mu \mathrm{m})\end{array}$ & $\begin{array}{c}\sigma \mathrm{z} \\
(\mu \mathrm{m})\end{array}$ \\
\hline $16 \mathrm{~mm}$ & 28 & 32 & 51 & 19 & 23 & 33 \\
\hline $12 \mathrm{~mm}$ & 16 & 13 & 28 & 13 & 12 & 22 \\
\hline $9 \mathrm{~mm}$ & 12 & 12 & 26 & 10 & 11 & 22 \\
\hline $4 \mathrm{~mm}$ & 8 & 7 & 16 & 10 & 8 & 18 \\
\hline $\begin{array}{c}\text { Mobile } \\
\text { phone }\end{array}$ & \multicolumn{3}{|l|}{$\mathrm{B}=1.8 \mathrm{~m}, \mathrm{H}=2.5 \mathrm{~m}$} & \multicolumn{3}{|c|}{$\mathrm{B}=3.0 \mathrm{~m}, \mathrm{H}=3.0 \mathrm{~m}$} \\
\hline $16 \mathrm{~mm}$ & 37 & 40 & 59 & 32 & 30 & 43 \\
\hline $12 \mathrm{~mm}$ & 22 & 25 & 37 & 18 & 21 & 31 \\
\hline $9 \mathrm{~mm}$ & 15 & 21 & 29 & 13 & 19 & 24 \\
\hline $4 \mathrm{~mm}$ & 18 & 24 & 30 & 24 & 27 & 32 \\
\hline
\end{tabular}

Table 5: Coordinate standard deviations under the different configurations.
Table 5 shows the standard deviations of the $\mathrm{x}, \mathrm{y}$ and $\mathrm{z}$ coordinates from the different configurations. In terms of the DSLR cameras, the best results are achieved through the use of the $4 \mathrm{~mm}$ target with the $2.5 \mathrm{~m}$ object distances. However, for the mobile phone camera station the best configuration was obtained through the $3.0 \mathrm{~m}$ object distance combined with $9 \mathrm{~mm}$ targets.

Compared with a DSLR camera, the focal length and image format of a mobile phone camera is much smaller, and these factors lead to the requirement for a larger target size. When the object distance was increased from $2.5 \mathrm{~m}$ to $3.0 \mathrm{~m}$, the use of $4 \mathrm{~mm}$ targets for the mobile phone-based imaging stations was found to be too small. Therefore, the photogrammetric software cannot automatically detect these $4 \mathrm{~mm}$ targets. This automatic process was replaced by manual selection and this substitution affected the precision. This may be the reason why the use of $4 \mathrm{~mm}$ targets has a lower precision in distance measurements than $9 \mathrm{~mm}$ targets.

\begin{tabular}{|c|c|c|c|c|c|c|}
\hline & \multicolumn{3}{|c|}{$\begin{array}{c}\mathrm{B}=1.8 \mathrm{~m}, \\
\mathrm{H}=2.5 \mathrm{~m}\end{array}$} & \multicolumn{3}{c|}{$\begin{array}{c}\mathrm{B}=3.0 \mathrm{~m}, \\
\mathrm{H}=3.0 \mathrm{~m}\end{array}$} \\
\hline Standard deviation $(\mu \mathrm{m})$ & $\sigma \mathrm{x}$ & $\sigma \mathrm{y}$ & $\sigma \mathrm{z}$ & $\sigma \mathrm{x}$ & $\sigma \mathrm{y}$ & $\sigma \mathrm{z}$ \\
\hline Targets on the platform & 16 & 23 & 27 & 15 & 21 & 35 \\
\hline $\begin{array}{c}\text { Targets on the moving } \\
\text { component }\end{array}$ & 18 & 25 & 30 & 16 & 23 & 32 \\
\hline
\end{tabular}

Table 6: Standard deviations of $9 \mathrm{~mm}$ targets based on different structural conditions.

Targets on the shaking table were divided into two different types. As Figure 5 illustrated, twenty-nine with 4 different size targets were attached onto the rigid platform and eleven of the twenty-nine targets on the platform used $9 \mathrm{~mm}$ targets. In addition, twelve $9 \mathrm{~mm}$ targets were arranged onto the non-fixed structure. Table 6 shows the comparison of these $9 \mathrm{~mm}$ targets. Although the results on two different structure conditions and two test field configurations have a few microns difference, these results are at the same level and are similar to the results shown in Table 5. The conclusion indicates that if the shutter speed is fast enough to freeze the structural deformation (i.e. the image is clear and sharp), the structure can be analysed as a still object. The structural movement therefore should not significantly reduce the measurement precision.

As mentioned above, there are nine sections that have been marked in Figure 5. The lengths of these sections were calculated using the photogrammetric results and the physical measurements individually. The mean of the physical measurement-based result was taken as a conventional true value and was used to compare the photogrammetric results to calculate a RMSE. Table 7 presents results generated from the mobile phone-based camera station data.

\begin{tabular}{|c|c|c|}
\hline \multirow{2}{*}{ Mobile phone camera } & $\mathrm{B}=1.8 \mathrm{~m}, \mathrm{H}=2.5 \mathrm{~m}$ & $\mathrm{~B}=3.0 \mathrm{~m}, \mathrm{H}=3.0 \mathrm{~m}$ \\
\cline { 2 - 3 } & $\mathrm{RMSE}(\mu \mathrm{m})$ & RMSE $(\mu \mathrm{m})$ \\
\hline 16mm target & 135 & 122 \\
\hline 12mm target & 79 & 73 \\
\hline 9mm target & 33 & 30 \\
\hline 4mm target & 39 & 35 \\
\hline
\end{tabular}

Table 7: RMSE of photogrammetric measurements. 
Under two different test field configurations, the $9 \mathrm{~mm}$ target has generated the optimal precision among the four different target sizes. The optimum performance occurred under the $3.0 \mathrm{~m}$ object distance configuration with $9 \mathrm{~mm}$ targets. In order to provide an indication idea of the precision of this mobile phone-based monitoring system, the RMSE for the $9 \mathrm{~mm}$ results (Table 7) can be divided by the longest physical measurement among the nine utilised sections $(753.342 \mathrm{~mm})$. This returns a precision of approximately 1:25,000. The results in Table 5 also indicate that the use of oversized targets will not increase the overall precision. Instead, reduced precisions are obtained.

\section{CONCLUSIONS AND FUTURE WORK}

The results in Table 2 indicated that this system is capable of handling a long-term, continuous monitoring project. Although individual image station crashes occurred, the automatic recovery function was able to re-start the image capture with only minor interruptions. And this automated recovery function can significantly improve the reliability for long-term monitoring purposes. Final results show that under good lighting conditions, with proper camera calibration, strong network configuration and the use of optimum target sizes, this low-cost, mobile phone-based monitoring system is capable of dealing with long-term, monitoring applications with a precision of approximately 1:25,000.

The on-going improvements to this system are focussing on software developments for enhancing the surveying functionality of mobile phone devices. The concept of this scheme aims to develop a new software application to allow civil engineers to perform basic photogrammetric processing using a single mobile phone (i.e. the mobile phone will be used to capture an image and then start to run procedures such as image processing, target detection, coordinate calculation etc.). Currently, a prototype has been developed. This software can generate good results, if targets are arranged onto a surface and provide a good contrast with the background. Different algorithms are being applied to improve the recognition rate. Also the minimization of system loading is another major concern. With the continued development of mobile phone hardware, and the ever improving quality of built-in cameras, better processing speeds and identification accuracy can be expected in the future.

\section{REFERENCES}

Akca, D., Gruen, A., 2009. Comparative geometric and radiometric evaluation of mobile phone and still video cameras. The Photogrammetric Record, 24 (127), pp. 217-245.

Foss, G.C., 2008. Photogrammetry of Aircraft Structures for Sensor Location. In: IMCA XXVI International Modal Analysis Conference. Orlando, USA, pp. 223-243.

Ganci, G., Clement, R., 2000. The use of self-identifying targeting for feature based measurement. In: Coordinate measuring system committee. Dearborn, Michigan, July 200. pp. 101-120.

Glisic, B., Inaudi, D., Ming, L.J., Yew, Y.T., Tat, N.C., 2007. Large Scale Lifespan Monitoring of High-Rise Buildings Using Long-Gage Fiber Optic Sensors. In: the 3rd International
Conference on Structural Health Monitoring of Intelligent Infrastructure. Vancouver, Canada. pp. 7.

González-Aguilera, D., Rodríguez-Gonzálvez, P., GómezLahoz, J., 2009. An automatic procedure for co-registration of terrestrial laser scanners and digital cameras. ISPRS Journal of Photogrammetry and Remote Sensing, 64 (3), pp. 308-316.

Instron, 2011. 3520 Series Hydraulic Power Units. http://plastics.informous.com/view/7813-3520-series-hydraulicpower-units-for-your-servohydraulic-test-systems/download (07 March 2012).

Kim, S., Pakzad, S.N., Culler, D., Demmel, J., Fenves, G.L., Glaser, S., Turon, M., 2006. Health Monitoring of Civil Infrastructures Using Wireless Sensor Network. In: the 4th International Conference on Embedded Networked Sensor Systems. New York, USA, pp. 254-263.

Lin, S.-Y., Mills, J.P., Gosling, P.D., 2008. Videogrammetric monitoring of as-built membrane roof structures. The Photogrammetric Record, 23 (122), pp. 128-147.

Luhmann, T., Robson, S., Kyle, S. and Harley, I., 2007. Close range photogrammetry: principles, techniques and applications. John Wiley \& Sons, New Jersey.

Maas, H.-G.a.H., U., 2006. Photogrammetric techniques in civil engineering material testing and structure monitoring. Photogrammetric Engineering \& Remote Sensing, 72 (1), pp. 39-46.

Mills, D.L., 1991. Internet time synchronization: the network time protocol. IEEE Transactions on Communications, 39 (10), pp. $1482-1493$.

Mills, D.L., 2006. Computer Network Time Synchronization: The Network Time Protocol. CRC Press.

Psimoulis, P., Stiros, S., 2007. Measurement of deflections and of oscillation frequencies of engineering structures using Robotic Theodolites (RTS). Engineering Structures, 29 (12), pp. 3312-3324.

Wang, C.-H., Mills, J.P., Gosling, P., Bridgens, B., Grisdale, R., 2010. Monitoring the testing, construction and as-built condition of membrane structures by close range photogrammetry. In: The International Archives of the Photogrammetry, Remote Sensing and Spatial Information Sciences, Newcastle, UK, Vol. 36, Part 5, pp. 592-596.

in: International Archives of Photogrammetry, Remote Sensing and Spatial Information Sciences. 2010, Newcastle, UK: ISPRS.

Young, L., Garde, G., 2007. A practical approach for scientific balloon film strain measurement using photogrammetry. In: AIAA Balloon Systems Conference. Williamsburg, VA, American Institute of Aeronautics and Astronautics, Inc, pp. 1117. 